Der Eingang zum Kongresshaus Biel.

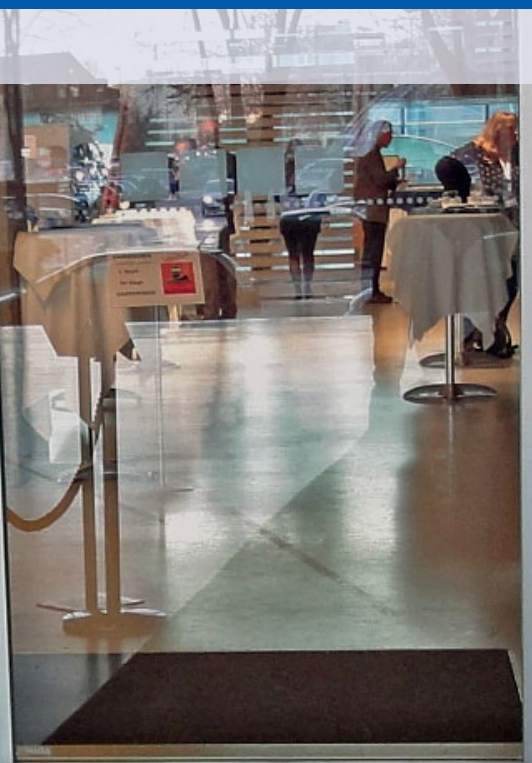

Fotos: Christoph Kreyden
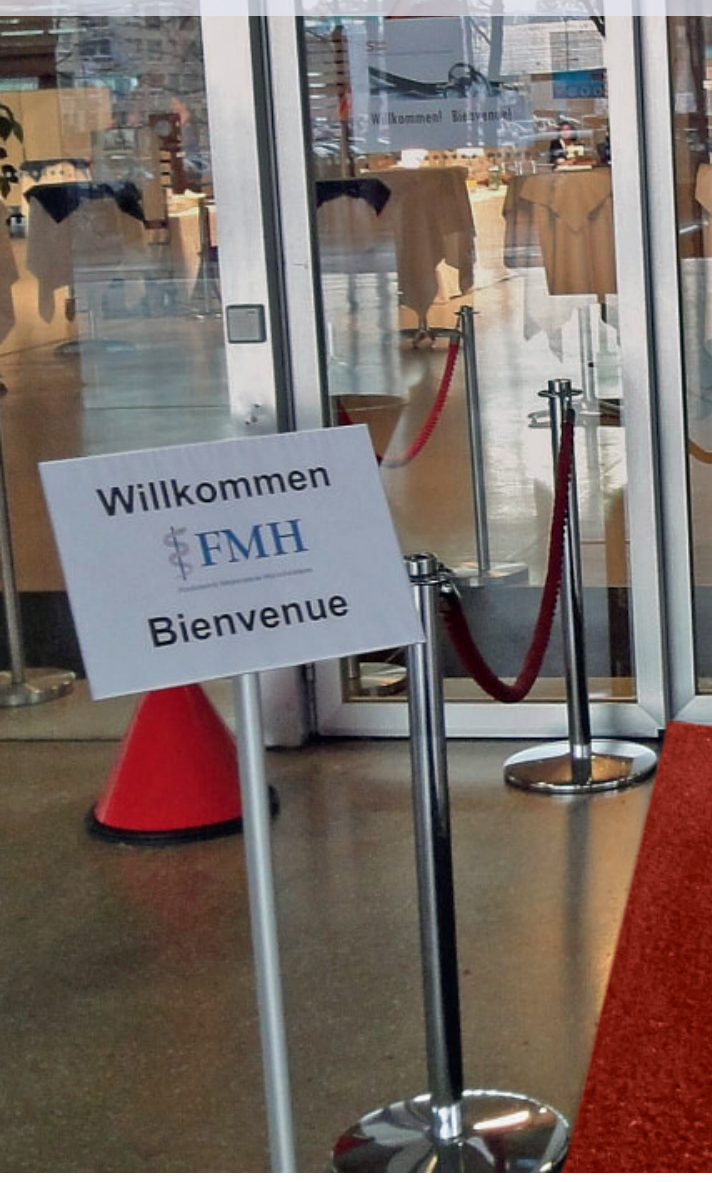

Kongresshaus, Biel, 10. Dezember 2009

\title{
Protokoll der ausserordentlichen Ärztekammer
}

Erika Flückiger,

Leiterin Dienste

Ein Glossar mit den am häufigsten verwendeten Abkürzungen finden Sie auf Seite 169.

Anmerkung der Protokollführerin: Zum besseren Verständnis werden die Geschäfte in der Reihenfolge der eingangs der Sitzung geänderten Traktandenliste protokolliert.

\section{Begrüssung, Mitteilungen, Bestellung des Büros}

Nach Erreichen des erforderlichen Quorums der Hälfte der stimmberechtigten Delegierten eröffnet der Präsident, Jacques de Haller, um 13.35 Uhr die ausserordentliche Sitzung der Ärztekammer. Hauptgeschäft dieser halbtägigen Sitzung ist die Wahl des Präsidenten des SIWF (Schweizerisches Institut für ärztliche Weiterund Fortbildung). Der Präsident begrüsst die anwesenden Delegierten und die Gäste: Hans Rudolf Koelz und Jean-Pierre Keller, die beiden Vizepräsidenten des SIWF, zwei Vertreter der Geschäftsprüfungskommission (GPK): Roland Schwarz und Adrian Sury, Bruno Kesseli, Chefredaktor der Schweizerischen Ärztezeitung (SÄZ), sowie Reinhard Kunz, Geschäftsführer der FMH Services.

Nach einigen organisatorischen Informationen bestellt der Präsident das Büro der heutigen Ärztekammersitzung. Es besteht aus dem FMH-Präsidenten, dem Vizepräsidenten Ernst Gähler (der zweite Vizepräsident Ignazio Cassis ist wegen der Sitzung des Nationalrates nicht anwesend), dem Generalsekretär und folgenden Stimmenzählern: Pierre Vallon/SGPP, Günther Gruber/Radio-Onkologie (SRO), Philippe Vuillemin/VD,
Tobias Ehmann/LU, Philipp Bertschinger/Gastroenterologie (SGG), Carlo Moll/VLSS, Britta Hafner/AI,AR, Urs von Wartburg/Handchirurgie (SGH), David U. Hutter/VSAO, Christoph Bosshard/VSAO.

Den Zeitpunkt, nach welchem keine Abstimmungen und Wahlen mehr durchgeführt werden können, legt der Präsident auf 17.30 Uhr fest.

\section{Traktandenliste}

Die Anträge, welche den Delegierten im Nachversand zugestellt wurden, sind diesen bei der Eingangskontrolle auch noch als Sitzungsunterlagen übergeben worden.

Christoph Bosshard/VSAO beantragt, Antrag Nr. $1 \mathrm{zu}$ Traktandum 7/Varia sei als eigenständiges Traktandum, über welches abgestimmt werden könne, aufzunehmen. Der Präsident lässt die Delegierten über die beantragte Änderung der Traktandenliste ( $2 / 3-$ Mehr notwendig) abstimmen. Dem Antrag wird mit grosser Mehrheit, bei 4 Gegenstimmen und einigen Enthaltungen, stattgegeben.

Der Präsident bittet anschliessend den Generalsekretär Daniel Herzog, den Delegierten die seit dem 1. Juni 2009 neu eingetretenen Mitarbeiterinnen und Mit- 
arbeiter vorzustellen. Es sind dies in der Reihenfolge ihres Anstellungsbeginns: Verena Schicker/Sachbearbeiterin DLM (Abt. Dienstleistungen, Mitgliedschaft), 1.6.2009; Sylvain Faÿsse, Leiter Übersetzungsdienst, 13. 7. 2009; Liliane Manz, Assistentin des FMHPräsidenten/Vorstandssekretariat, 1.8.2009; Myriam Möschinger, Sekretariat Tarifdienst, 1.8.2009; Mariko Küng, Sekretariat Rechtsdienst und Bereich MPA (Med. Praxisassistentinnen), 1.10.2009; Varja Nikolic, wissenschaftliche Mitarbeiterin DDQ (Abt. Daten, Demographie und Qualität), 1.10.2009; Susanne Christen, Ärztin, Tarifdienst/Bereich Tarife und Verträge, 16.11.2009.

\section{Wahl des SIWF-Präsidenten}

Der Präsident informiert, dass zu diesen Traktandum von diversen Organisationen (Jürg Nadig/SGMO und SFSM, Christoph Bosshard/VSAO, Urban Laffer/fmCh, Christoph Ramstein/VEDAG, Werner Bauer/SGIM) ähnlich lautende Anträge eingegangen sind, welche im Antrag Nr. 1 zu Traktandum 2 zusammengefasst sind. Die Antragsteller verlangen, «dass die Ärztekammer vor der Wahl des SIWF-Präsidenten, die Regelungen im Geschäftsreglement des SIWF bestätigt, wonach die GPK für die Anstellungsbedingungen des SIWF-Präsidenten, der SIWF-Vorstand für diejenigen des Geschäftsführers, zuständig ist. Dass der ZV beauftragt werde, für eine Verankerung dieser und allenfalls weiterer Regelungen in Statuten und Geschäftsordnung der FMH zu sorgen, um ein einwandfreies und unabhängiges Funktionieren des SIWF sicherzustellen sowie, dass der ZV die vorgeschlagenen Statuten- und GO-Änderungen an der Delegiertenversammlung (DV) vom 11. Februar 2010 vorstelle und der Ärztekammer am 27. Mai 2010 zum Beschluss vorlege».
Der Präsident hält fest, der Zentralvorstand sei bereit, diesen Antrag entgegenzunehmen, allerdings mit folgenden Nuancen: Formell hat die Ärztekammer keine Kompetenz, das Geschäftsreglement des SIWF zu bestätigen, diese Kompetenz liegt allein beim SIWFPlenum. Den verlangten Termin vom 11. Februar 2010 erachtet der Zentralvorstand als zu früh. Der neue SIWF-Präsident soll unbedingt in die Diskussionen einbezogen werden, eine Vorlage der geplanten Statuten- und GO-Änderungen an der DV vom März 2010 sei terminlich eher machbar und früh genug, um das Geschäft zu diskutieren und der ordentlichen Ärztekammer vom Mai 2010 einen Antrag zum Beschluss vorzulegen.

J. Nadig/SGMO und SFSM sowie Ch. Bosshard/ VSAO halten an einer Vorlage im Sinne einer ersten Lesung an der DV vom 11. Februar 2010 und damit an ihrem Antrag ohne die vom Präsidenten formulierten Nuancen fest. Hanspeter Kuhn/Stv. Generalsekretär präzisiert zuhanden des Protokolls, dass die Annahme des Antrags für den ersten Antragspunkt (Bestätigung des SIWF-Geschäftsreglementes) nicht eine Genehmigung bedeuten könne, sondern als politische Äusserung zu verstehen wäre. Marc Müller/ KHM bekräftigt, dass gerade die Annahme dieses Antragspunktes ein starkes Signal seitens der Ärztekammer für die Unabhängigkeit des SIWF darstellen würde.

Nachdem der Präsident nochmals nachdrücklich festhält, dass der ZV mit den Grundsätzen des Antrags einverstanden sei, ersucht er die Delegierten, darüber abzustimmen.

Die Ärztekammer genehmigt den Antrag Nr. $1 \mathrm{zu}$ Traktandum 2 einstimmig, bei 5 Enthaltungen.

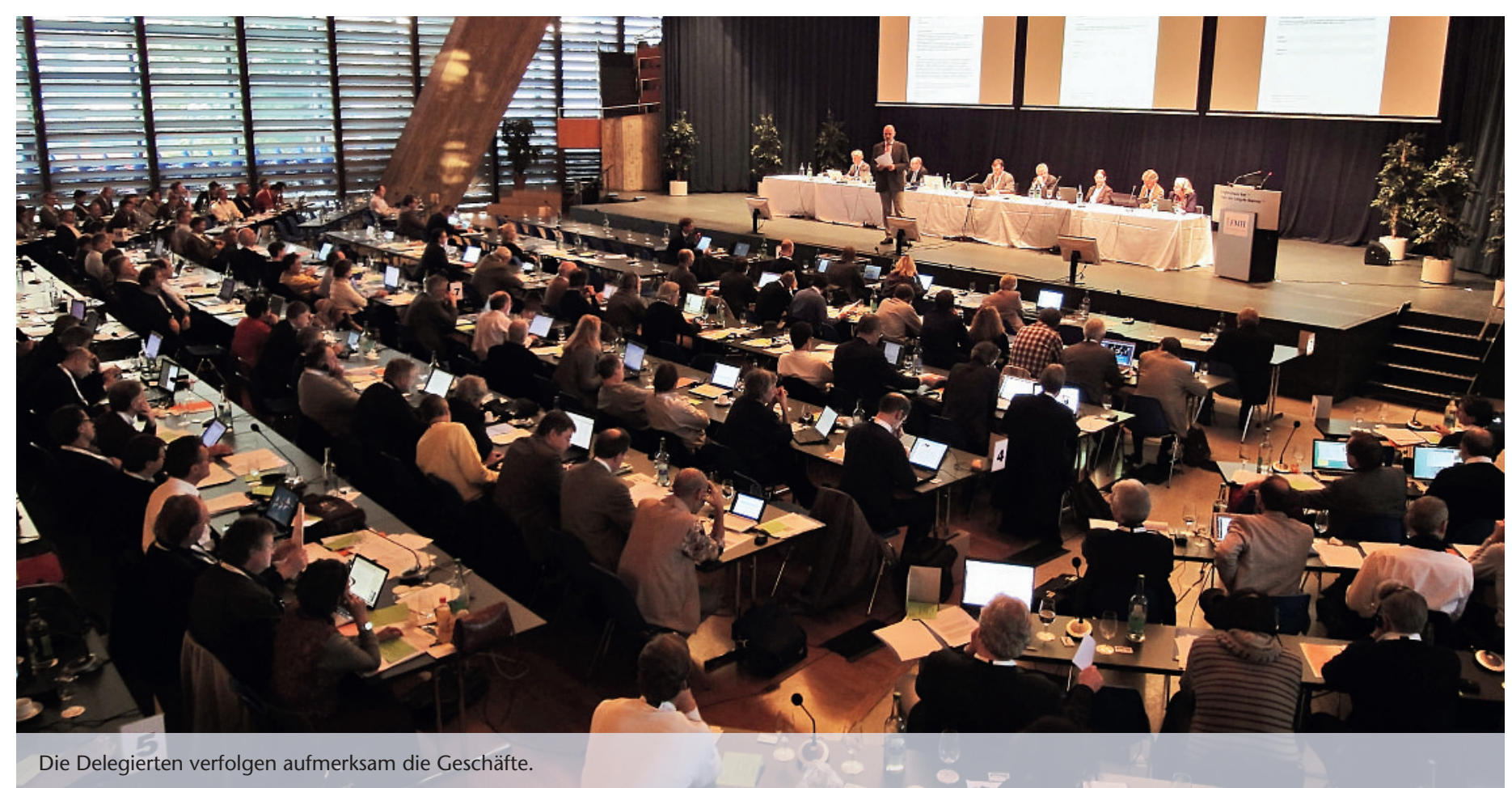


An dieser Stelle ergreift Max Giger/Präsident SIWF und $Z$ V-Mitglied das Wort, um zu erklären, weshalb er nochmals für das Amt kandidiert: Vor dem Hintergrund des Bestrebens, das SIWF solle stark nach aussen auftreten, erachtet er das Findungsprozedere, aus welchem schliesslich nur noch ein einziger Kandidat resultierte, als ungenügend. Er stehe deshalb noch für eine weitere Zeit zur Verfügung, während welcher nochmals eine Findung erfolgen soll, bekräftigt aber gleichzeitig, dass er sich ausdrücklich weder gegen den aus dem Findungsprozedere hervorgegangenen Kandidaten noch gegen den Kandidaten stelle, welcher sich nachträglich gemeldet hat. Deshalb würde er auch seine Kandidatur zurückziehen, falls sich die Ärztekammer gegen ein neues Findungsprozedere ausspreche.

Dem Vorschlag des Präsidenten, jetzt über Eintreten in das Wahlprozedere des SIWF-Präsidenten abzustimmen - wobei ein Nein das Hinausschieben der Wahl bedeuten würde - erwächst Widerstand seitens C. Moll/VLSS und Th. Keller/SZ. Ph. Vuillemin/VD stört sich daran, dass M. Giger in autoritärer Art das Wort ergriffen hat, und äussert sich kritisch dazu, dass jetzt seit der Verlängerung der Amtsdauer von M. Giger, die dazu dienen sollte, dessen Nachfolger zu finden und einzuarbeiten, 18 Monate verstrichen seien. Er spricht sich klar gegen eine nochmalige unbefristete Weiterführung des Mandates von M. Giger aus. Alle drei Redner verlangen, dass der Vorsitzende der Findungskommission der Ärztekammer nun seinen Bericht vorlegt.

Der Präsident erteilt das Wort dem Vorsitzenden der Findungskommission, Dieter Conen, welcher den Ärztekammer-Delegierten den Bericht über das Findungsprozedere präsentiert:

Auf Wunsch des SIWF-Vorstandes hat die Delegiertenversammlung (DV) im September 2009 beschlossen, das ab 1. Juni 2010 frei werdende Amt des SIWFPräsidenten öffentlich auszuschreiben. Gleichzeitig wurde eine Findungskommission mit dem Auftrag eingesetzt, geeignete Kandidatinnen/Kandidaten zuhanden der heutigen ausserordentlichen Ärztekammer zu evaluieren. Der Kommission gehörten folgende Vertreter-innen der Dachverbände an: Werner Bauer/KHM, Jean Biaggi/fmCh, Daniel Bielinski/FMPP, CharlesAbram Favrod-Coune/SMSR, Claudia Ferrier-Guerra/ OMCT, Carlo Moll/VLSS, Jürg Nadig/SFSM, Christoph Ramstein/VEDAG, Raphael Stolz/VSAO, Albert Urwyler/Vertreter med. Fakultäten sowie als Gäste: Jacques de Haller/FMH-Präsident, Hans Rudolf Koelz/Vizepräsident SIWF. An ihrer ersten Sitzung definierte die Kommission das Anforderungsprofil, das Inserat erschien in der SÄZ Nr. 38. Von den insgesamt 6 Kandidierenden (5 Kandidaten, 1 Kandidatin) lud die Findungskommission 3 Kandidaten und 1 Kandidatin für ein ausführliches Interview ein. Aus der Evaluation gingen schliesslich zwei Kandidaten hervor, welche die Findungskommission der a.o. Ärztekammer von heute vorschlagen wollte. Einer der beiden Kandidaten hat sich Ende November 2009 zurückgezogen, so dass als einziger Kandidat, der das Findungsprozedere durchlaufen hat und von der Findungskommission zur Wahl empfohlen wird, Pierre-François Cuénoud, aktuell Mitglied des Zentralvorstands, verbleibt. Inzwischen hat Werner Bauer seine Kandidatur angemeldet.

Die sehr detaillierte Schilderung des Vorsitzenden der Findungskommission, wie die Interviews mit den einzelnen Kandidaten/Kandidatin - unter voller Namensnennung - abliefen, führt zu einem vehementen Einspruch durch Ch. Bossard/VSAO, der verlangt, entweder auf diesen Teil des Berichtes ganz zu verzichten oder aber zumindest die Namen der Kandidierenden, die nicht in die engere Wahl kamen, abzudecken. Die im Saal anwesende Kandidatin übt erhebliche Kritik an der unprofessionellen Art und Weise, wie die Findungskommission das Interview durchgeführt habe.

Im Anschluss an den Bericht stellt der Präsident nochmals die Frage, ob ein Antrag auf Nichteintreten gestellt werde. Dies ist nicht der Fall, weshalb Eintreten auf den Wahlvorgang als beschlossen zu bezeichnen ist. Auf die Frage des Präsidenten an M. Giger, ob er nun kandidiere oder nicht, bestätigt dieser, er sei nicht Kandidat.

Der Präsident erläutert, es entspreche unseren Statuten, dass bis zum Beginn des Wahlvorgangs neben den beiden jetzt bekannten Kandidaten P.-F. Cuénoud und W. Bauer noch jeder und jede Interessierte kandidieren könne, und erkundigt sich, ob noch weitere Anwesende für das Amt kandidieren möchten. Das scheint nicht der Fall zu sein. Dieses Vorgehen stösst bei verschiedenen Delegierten, insbesondere aber beim VLSS, auf Kritik. C. Moll und Hansueli Würsten finden die Kandidatur von W. Bauer gegenüber dem Kandidaten P.-F. Cuénoud, welcher das ganze Findungsprozedere habe durchlaufen müssen, nicht korrekt. H. Würsten ist der Meinung, dass nur P.-F. Cuénoud zur Wahl gestellt werden solle und, sollte er nicht gewählt werden, der ganze Prozess wieder von vorne beginnen müsse.

Der Präsident wiederholt nochmals, dass das Vorgehen demokratisch sei, dass man über die Eignung des einen oder andern Kandidaten anschliessend, in deren Abwesenheit, diskutieren könne. Wenn die Ärztekammer anderer Meinung sei, erwarte er jetzt einen Ordnungsantrag. Ein solcher wird nicht eingereicht. Der Präsident weist darauf hin, dass den Delegierten die Unterlagen zu den Kandidaturen im Nachversand zugestellt wurden, und schlägt vor, jetzt die beiden Kandidaten anzuhören. Er erteilt, der alphabetischen Reihenfolge entsprechend, zunächst W. Bauer das Wort.

Werner Bauer erklärt, er sehe sich keineswegs als Sprengkandidat. Als Mitglied der Findungskommission habe er deren Arbeit miterlebt und erachte diese als seriös. Schliesslich sei aber nur noch ein einziger Kandidat übriggeblieben. Von verschiedenen Seiten sei man dann an ihn herangetreten mit der Frage, ob er sich nicht vorstellen könne, dieses Amt zu über- 
nehmen. Dann habe er sich von der Idee begeistern lassen. Über ein langes Wochenende habe er sich darüber Gedanken gemacht und würde die Aufgabe nun mit Freude angehen. Er streift kurz einige Punkte in seinem Lebenslauf und kommt dann ausführlicher auf seine Ziele zu sprechen, die er sich als SIWF-Präsident stecken würde: Die Weiter- und Fortbildung als Kernaufgabe der ärztlichen Berufspolitik muss verschiedenste Anliegen berücksichtigen: strukturierte Weiterbildungsgänge für die junge Generation, Anforderungen von Patienten und Öffentlichkeit, Förderung einer hohen Arbeitsqualität und Vermeiden von Überregulierungen. Den Einfluss der Ärzteschaft diesbezüglich einzuschränken, Regelsetzungen und Verantwortung dafür andern Akteuren zu übertragen, ist weltweit Tendenz, weil - so wird argumentiert - eine Verflechtung von materiellen Interessen und Federführung für Weiter- und Fortbildung durch die gleiche Ärzteorganisation nicht akzeptabel sei. Er möchte deshalb das folgerichtig gegründete Schweizerische Institut für Weiter- und Fortbildung/SIWF mitgestalten und als unabhängige Institution, in welchem öffentlichen Institutionen die Möglichkeit zur Mitwirkung gegeben wird, fest im Gesundheitswesen verankern. Er möchte dem «Teaching» in der Schweiz zu einer Aufwertung verhelfen und, ähnlich der in vor allem angelsächsischen Ländern bestehenden Tradition der «Colleges of Physicians» als ärztlich geprägten und hochangesehenen Institutionen für Weiter- und Fortbildung, eine schweizerische Variante zu schaffen versuchen. Für eine strukturierte, modulare, finanziell gesicherte Weiterbildung, in der die Zukunft unserer Ärzteschaft liegt, sowie für eine sinnvolle, auf der Selbstverantwortung aufbauende Fortbildung, in der die Qualität der ärztlichen Arbeit liegt, möchte er sich in den nächsten Jahre mit Begeisterung einsetzen.

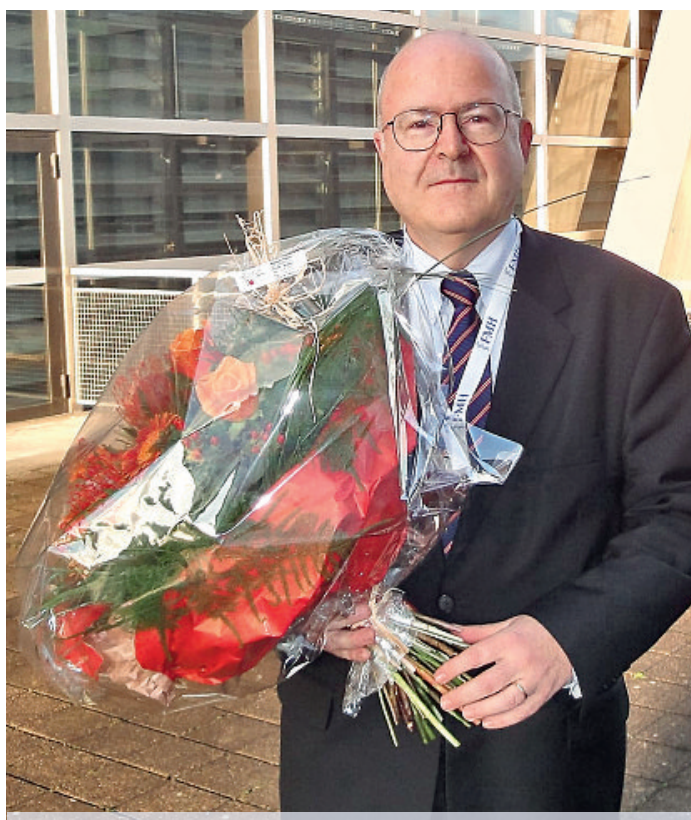

Der neue Präsident des SIWF: Dr. med. Werner Bauer.
Der Präsident erteilt das Wort anschliessend an P.-F. Cuénoud. Pierre-François Cuénoud erklärt eingangs, dass die Ausbildung des Nachwuchses seit jeher seine Hauptmotivation war, die den roten Faden in seiner nun 9-jährigen Tätigkeit als Leiter einer chirurgischen Weiterbildungsstätte bilde, und dass die Ausschreibung klar Erfahrung in der Lehre verlange. Er möchte aber weniger auf seine schriftlich vorliegende Kandidatur eingehen, sondern vielmehr auf die Frage antworten, ob ein aktuelles Mitglied des Zentralvorstandes fähig sei, die Unabhängigkeit zu demonstrieren. Er würde, im Falle seiner Wahl, sofort aus dem Zentralvorstand zurücktreten. Auch innerhalb des ZV habe er sein Ressort immer eigenständig geführt. SwissDRG sei nun gut aufgegleist, verfüge über ein Team von sehr fähigen Mitarbeitenden, die eine hohe Arbeitsqualität leisteten, und es bleibe genügend Zeit, einen Nachfolger zu suchen. Er würde sich gerne dieser neuen Herausforderung stellen und mit viel Engagement in den nächsten 10 Jahren eine überzeugende Vision für die ärztliche Weiter- und Fortbildung entwickeln, seine Kenntnisse, Beziehungen und Erfahrung in den Dienst des neuen SIWF stellen, um diesem eine gebührende Position gegenüber dem Bund, den Kantonen, den Universitäten und den Spitälern zu sichern.

Nachdem die beiden Kandidaten auf Bitte des Präsidenten den Saal verlassen haben, erklärt der Präsident die Diskussion als eröffnet: Verschiedene Redner heben die Vorteile des einen gegenüber dem andern Kandidaten hervor: die einen machen sich stark für einen Spitalarzt, da die Weiterbildung doch zu 90\% in den Spitälern stattfinde, die andern heben die hohe Sachkenntnis in Weiter- und Fortbildungsfragen als wichtig hervor. Es wird auch argumentiert, dass P.-F. Cuénoud als ZV-Ressortleiter SwissDRG in der Öffentlichkeit stark mit Finanzen und Tarifen verankert sei, man kenne ihn als «Mr. DRG», was dem Bestreben, die Unabhängigkeit des SIWF zu stärken, abträglich sein könnte. Andere Redner würden bedauern, im Ressort SwissDRG jetzt so kurz vor der geplanten Einführung der DRGs einen Stabwechsel durchführen zu müssen. Einhellig sind die Delegierten der Meinung, es stünden hier zwei hervorragende Kandidaten zur Wahl. Schliesslich bringen es verschiedene Delegierte auf den Punkt: Die beiden Kandidaten nützen der FMH am meisten, wenn P.-F. Cuénoud im ZV verbleibt und das Ressort SwissDRG dort weiter betreut und W. Bauer heute zum SIWF-Präsidenten gewählt wird. Die beiden hervorragenden Kandidaten an diesen beiden Schlüsselstellen zu haben, würde der FMH zu einer starken Position verhelfen.

Nachdem das Wort nicht mehr verlangt wird, hält der Präsident ausdrücklich fest, dass der Zentralvorstand, welcher über Mittag die Ausgangslage nochmals kurz diskutiert hatte, der einhelligen Ansicht ist, mit beiden Kandidaten sehr gut zusammenarbeiten zu können. Er erläutert anschliessend das Wahlprozedere: Für die Wahl ist das absolute Mehr nötig, leere Wahlzettel sind gültig, sie zählen für die Ermittlung des 
absoluten Mehrs. Der Präsident bittet die Stimmenzähler, die Wahlzettel einzusammeln und die Urnen ins Wahlbüro zu bringen, wo die Stimmen durch Mitarbeitende des FMH-Generalsekretariates unter Leitung von Christoph Kreyden ausgezählt werden.

(Während der Auszählung erteilt der Präsident das Wort an Daniel Herren für die Informationen aus seinem Ressort DDQ Traktandum 5.1.)

Der Präsident verkündet anschliessend das Resultat des 1. Wahlgangs: eingegangene Stimmzettel: 165; gültig: 162; Enthaltungen: 3; ungültige Stimmzettel: 0; total gültige Stimmzettel: 165, absolutes Mehr: 83. Zum neuen SIWF-Präsidenten gewählt ist mit 143 Stimmen: Werner Bauer

Der Präsident Jacques de Haller gratuliert W. Bauer herzlich zur Wahl und zur breiten Abstützung in der Ärztekammer. Letztere spendet dem Gewählten einen langen und warmen Applaus. W. Bauer bedankt sich herzlich und zeigt sich gerührt und bewegt. Es sei ihm sehr wichtig, die breite Unterstützung der Ärztekammer zu haben, und er freue sich auf die Zusammenarbeit mit den verschiedenen Gremien. Abschliessend drückt er Max Giger und seiner Crew seinen grossen Dank aus.

\section{Statuten- und Geschäftsordnungs-} Änderungen, Nachführen der Standesordnung

Aufnahme der Schweizerischen Ärztegesellschaft für Manuelle Medizin (SAMM) in die Schweizerische Ärztekammer als mitspracheberechtigte Ärzteorganisation

Der Zentralvorstand beantragt der Ärztekammer die Aufnahme der Schweizerischen Ärztegesellschaft für Manuelle Medizin als mitspracheberechtigte Organisation gemäss Art. 25 Abs. 2 der FMH-Statuten.

Das Wort dazu wird nicht verlangt. Der Präsident bringt den Antrag zur Abstimmung.

Die Ärztekammer genehmigt den Antrag Nr. 1 zu Traktandum Nr. 3.1 mit grossem Mehr, einer Gegenstimme und 8 Enthaltungen. Anhang III der FMH-Statuten wird mit der Schweizerischen Ärztegesellschaft für Manuelle Medizin ergänzt.

\section{Information über das Salärsystem}

für den Zentralvorstand

Die Ärztekammer hatte an ihrer ordentlichen Sitzung vom 28. Mai 2009 aufgrund eines von Ch. Ramstein/ VEDAG eingereichten Antrages den Zentralvorstand beauftragt, einen Vorschlag zur Umsetzung des de facto Postulates «Offenlegung ZV-Saläre» auszuarbeiten. Der Präsident erläutert, dass die Überlegungen des Zentralvorstandes zwei Ziele anvisierten: die Transparenz gegenüber geeigneten FMH-Organen und die Vermeidung von Missverständnissen gegen aussen. Der Zentralvorstand sei zum Schluss gekommen, das geeignete Organ zur «Offenlegung der ZV-Saläre» sei die Delegiertenversammlung. Deren überschaubare Grösse eigne sich, um die Thematik diskutieren und allfällige Fragen zu klären. Die Delegiertenversammlung (DV) habe diesen Vorschlag an ihrer Sitzung vom 10. September 2009 genehmigt. Die Geschäftsprüfungskommission (GPK) befürworte die vorgeschlagene Lösung ebenfalls.

Der Zentralvorstand unterbreitet deshalb der Ärztekammer den Antrag Nr. 1 zu Traktandum 3.2., wonach er die DV über das von der GPK für den ZV festgelegte Salärsystem informiert. Diese Informationen sollen in der Regel weder veröffentlicht noch protokolliert werden.

Diesem Antrag erwächst seitens des VSAO und der Ärztegesellschaft des Kantons Bern Widerstand. Ch. Bosshard erläutert den Antrag des VSAO, Nr. 2 zu Traktandum 3.2: Der VSAO zeigt sich damit einverstanden, dass der ZV die DV über das von der GPK für den ZV festgelegte Salärsystem informiert. Zusätzlich soll aber die Gesamtsumme aller Bezüge der ZVMitglieder alljährlich allen Ärztekammerdelegierten schriftlich mitgeteilt und die höchste durch ein Mitglied bezogene Summe soll gesondert ausgewiesen werden.

Jürg Schlup/BE erläutert den Antrag der Berner Ärztegesellschaft, welcher eine Information lediglich des Systems (und nicht der effektiven Zahlen) der Saläre, wie es der ZV vorschlägt, nicht zu genügen vermag. Die Berner Ärztegesellschaft informiere jährlich individualisiert über die Salärbezüge. Ihr Antrag will deshalb, dass die Jahresbezüge der einzelnen Mitglieder des ZV den Ärztekammerdelegierten alljährlich schriftlich mitgeteilt werden.

Der Präsident eröffnet die Diskussion, das Wort wird jedoch nicht verlangt. Er erläutert deshalb noch einmal den ZV-Antrag. Der ZV finde es richtig, dass seine effektiven Saläre bekannt seien. In der DV seien alle Dachverbände vertreten. Diskutiere aber die Ärztekammer über die ZV-Saläre, werde dies protokolliert, das Ärztekammerprotokoll erscheine in der SÄZ, welche überall gelesen werde. Er erachte es als unnötig, in der Öffentlichkeit Diskussionen heraufzubeschwören, die, gerade wenn es um Saläre gehe, ohnehin meist zu Missverständnissen führten. Der ZV sei klar für Transparenz, dies jedoch gegenüber der DV, in welcher alle vertreten seien.

J. Schlup/BE präzisiert noch einmal, dass seine Gesellschaft nicht damit einverstanden sei, dass der ZV nur das System, nicht aber die Saläre selbst offenlegen und er diese Information nur mündlich, statt schriftlich geben wolle

Der Präsident wiederholt, dass mit Salärsystem die Salärstufen gemeint seien. Ch. Ramstein/VEDAG erklärt, als eigentlicher Urheber dieses Antrages genügten ihm die Erläuterungen des Präsidenten, sein Antrag sei damit erfüllt. J. Schlup/BE verlangt vom Präsidenten in diesem Fall eine präzisierte Formulierung im ZV-Antrag, die im Protokoll festzuhalten sei. D. Herzog/ Generalsekretär schlägt vor, das Wort «Salärsystem» durch «Salärsystem inkl. Salärhöhen» zu ersetzen.

J. Schlup/BE hält aber an seinem Antrag fest. 
Der Präsident lässt die Ärztekammer zunächst über den von J. Schlup/BE eingereichten Antrag Nr. 3 zu Traktandum 3.2 abstimmen.

Die Ärztekammer genehmigt diesen Antrag mit 72 zu 49 Stimmen, ohne Enthaltungen.

Ch. Bosshard/VSAO zieht daraufhin seinen Antrag Nr. 2 zu Traktandum 3.2 zurück.

Der Präsident stellt in einer weiteren Abstimmung Antrag Nr. 3/J. Schlup/BE dem Antrag Nr. 1/ZV gegenüber.

Die Ärztekammer spricht sich mit 88 Stimmen für den von J. Schlup/BE eingereichten Antrag Nr. 3 zu Traktandum 3.2 aus. Der Antrag des ZV, Nr. 1 zu Traktandum 3.2 unterliegt mit 59 Stimmen.

\section{Standards für Telekonsultationszentren: \\ Ergänzung Standesordnung}

Hanspeter Kuhn/Stv. Generalsekretär erläutert den Antrag des Zentralvorstands Nr. 1a zu Traktandum 3.3. Das Bedürfnis, schweizerische Standards für Telekonsultationszentren zu erarbeiten, bestand schon seit einiger Zeit. Beschleunigt wurde das Thema durch die dringliche KVG-Revision von Bundesrat Couchepin (Telefonberatung als obligatorisches Angebot der Versicherer). Eine Arbeitsgruppe, in welcher insbesondere Vertreter der Schweizerischen Gesellschaft für Telemedizin und eHealth mitarbeiteten, hat einen Entwurf solcher Standards erarbeitet. Die Tatsache, dass seit September 2009 ein Internationaler Entwurf für solche Standards vorlag, vereinfachte die Aufgabe. Die DV vom 18. November 2009 befand, eine Trennung zwischen Telekonsultationszentren und den Telefonzentralen für den ärztlichen Notfalldienst sei in der Realität nicht mehr so klar möglich. Der Ärztekammer solle ein möglichst kurzer Passus als Ergänzung der Standesordnung vorgelegt werden. Die Standards selbst müssten überarbeitet und der DV in einem späteren Zeitpunkt nochmals unterbreitet werden. Die bestehende Arbeitsgruppe sei durch einen Vertreter der standeseigenen Einrichtungen für den Notfalldienst zu ergänzen.

Der Antrag Nr. 1a des Zentralvorstands schlägt folgenden Art. 33ter vor: «Institutionen, die den Patienten medizinische Telekonsultationen anbieten, sind Teil der Medizin. Sie verfügen über eine Betriebs- und/oder Berufsausübungsbewilligung und eine angemessene Haftpflichtversicherung. Sie sind finanziell, organisatorisch, personell und räumlich unabhängig von Unternehmen der Heilmittelindustrie (Pharma- und Medizinprodukteunternehmen) und Versicherungen (Krankenkassen, andere Sozial- und Privatversicherer). Sie beachten die Schweizer Standards für Telekonsultationszentren.»

J. Schlup/BE begründet seinen Antrag Nr. 2 zu Traktandum 3.3 damit, dass man sich durch die Formulierung des neuen Artikels 33ter nicht unnötig einschränken wolle, denn nicht-ärztliche Callcenter hätten sehr wohl Absprachen, wie sie der ZV-Vorschlag ausschliessen wolle, und sie seien abhängig von Versicherungsleistungen. Auf dem Markt hätten die standeseige- nen Einrichtungen damit ungleiche Spiesse, es würde ihnen die Möglichkeit genommen, mit Versicherern finanzielle Abmachungen zu treffen. Er möchte den Text auch deshalb kürzen, weil der Anhang - die Standards - noch unfertig sei. T. Heuberger/BE plädiert für den vereinfachten Berner Antrag: In der Standesordnung sei nur der Grundsatz zu formulieren, Ausführungen bzw. Standards gehörten in den Anhang, der ebenso verpflichtend für alle sei. Charles-Abram FavrodCoune/VD möchte einen Wunsch äussern, der weder am ZV-Vorschlag noch am Berner Antrag, den er allerdings vorzieht, etwas ändert. Callcenters oder wie man sie auch immer nennen möge, seien eine absolut wichtige Einrichtung, vor allem für Notfalldienst leistende Ärzte, insbesondere in der Peripherie, wo eine effiziente Triage sicher wünschbar sei. Für diese Ärzte wäre es ausserordentlich schade, wenn sich solche Einrichtungen nicht entwickeln könnten. Er erwähnt als Beispiel den Kanton Wallis, einen Bergkanton, der zu wenig Ärzte hat und der das Problem des Notfalldienstes nur dank eines sehr intelligent aufgezogenen Telefoncenters lösen konnte. Damit wolle er nicht sagen, alle Telefoncenter sollten nach dem Beispiel des Kantons Wallis organisiert werden, aber hier sei etwas sehr Gutes entstanden. Er habe etwas erstaunt gelesen, dass die Arbeitsgruppe mit Ärzten aus ärzteeigenen Notfalldienstzentren ergänzt werden soll. Sein Wunsch ist, dass für die Arbeitsgruppe auch Ärzte aus der Peripherie beigezogen werden, genauso aber auch Ärzte aus Städten, in denen, in Anführungszeichen, eine Plethora von Grundversorgern herrsche. Laut Präsident kann diesem Wunsch ohne Probleme entsprochen werden. Der Präsident erteilt das Wort anschliessend an Hugo Kupferschmidt/SGKPT für seinen Antrag Nr. 3 zu Traktandum 3.3, mit welchem folgende Ergänzung des ZV-Antrags verlangt wird, sollte dieser angenommen werden: «Das Schweizerische Toxikologische Informationszentrum gilt nicht als Telekonsultationszentrum im Sinne dieses Artikels.» Sollte der kurze Text gemäss Antrag J. Schlup/BE angenommen werden, würde der Antrag der SGKPT hinfällig. H. Kupferschmidt erläutert, dass die Tätigkeit des Toxzentrums von den Standards direkt betroffen und mit diesen, nach dem Wortlaut, wie er im Entwurf vorliegt, nicht kompatibel ist. Auch sei das Toxzentrum weder bei der Formulierung des Artikels noch der Ausarbeitung der Standards konsultiert worden, woraus er folgert, man habe nicht an das Toxzentrum gedacht. Hp. Kuhn/Stv. Generalsekretär bestätigt, dass in der Tat niemand daran gedacht hat. Sowohl die Diskussionen in der DV als auch heute in der Ärztekammer zeigten aber klar auf, dass die Standards so umformuliert werden müssten, dass sie nicht nur auf Telekonsultationszentren, die hier ihren Businesscase haben, anwendbar sind, sondern auch die Notfallmedizin sinnvoll abdecken. In den Überlegungen, welche Regeln für welche Institutionen passen, werde man ohne Probleme eine sinnvolle Regelung für das Toxzentrum finden. H. Kupferschmidt/ SGKPT schlägt vor, dass zunächst über den Antrag von 
J. Schlup/BE abgestimmt wird, weil sich je nach Ergebnis sein Antrag allenfalls damit erledige.

Der Präsident kommt diesem Wunsch nach und legt den Antrag J. Schlup/BE Nr. 2 zu Traktandum 3.3 mit dem Wortlaut: «Institutionen, die den Patienten medizinische Telekonsultationen anbieten, sind Teil der Medizin. Diese Institutionen müssen Standards einhalten, die in einem Anhang definiert werden», zur Abstimmung vor. Die Ärztekammer stimmt diesem Antrag mit grossem Mehr, bei zwei Gegenstimmen und 5 Enthaltungen, $z u$. H. Kupferschmidt/SGKPT zieht daraufhin, wie angekündigt, seinen Antrag Nr. 3 zu Traktandum 3.3 zurück.

\section{Bestätigung für \\ die FMH-Delegiertenversammlung}

Der Ärztekammer liegt ein Antrag auf folgende Mutationen in der Delegiertenversammlung vor:

Ordentliche Delegierte: FMPP: Hélène Beutler, Moutier, für den zurücktretenden Jürg Unger, Zürich Ersatzdelegierte: FMPP: Christian Bernath, Thalwil, für den zurücktretenden Giampiero Enderli, Locarno.

Nachdem das Wort nicht verlangt wird, bringt der Präsident den Antrag Nr. 1 zu Traktandum 4 des Zentralvorstands zur Abstimmung.

Die Ärztekammer bestätigt einstimmig die beantragten Mutationen.

\section{Informationen aus den Ressorts}

\section{Qualitätsstrategie des Bundes und Vereinbarung} Qualitäts-Kommission mit santésuisse

(Diese Informationen erfolgten während der Auszählung der Stimmen unter Traktandum 2/Wahl des SIWF-Präsidenten.)

Daniel Herren/Ressortleiter DDQ im Zentralvorstand informiert im Folgenden die Delegierten über zwei aktuelle Geschäfte aus seinem Ressort. Zunächst zur Qualitätsstrategie des Bundes, die nach einer recht langen Wartezeit endlich publiziert wurde. Kurz zusammengefasst der Inhalt dieser Strategie, die Ziele des Bundes und, daraus folgend, die Konsequenzen für die FMH bzw. das Ressort DDQ: Das Q-Strategiepapier beschreibt als Vision des Bundes eine hohe Qualität in der ambulanten und stationären Gesundheitsversorgung zu angemessenen und tragbaren Kosten, die führende Rolle des Bundes in der Qualitätssicherung sowie eine klare und eindeutige Rollenverteilung, welche die Zuständigkeiten, Verantwortungsbereiche und die Koordination zwischen den Akteuren (Bund - Kantone - Versicherer - Leistungserbringer - Patienten andere) regelt.

Zielsetzungen des Bundes sind, die Qualität im schweizerischen Gesundheitswesen laufend, nachhaltig und nachweislich zu verbessern. Dabei sollen die Massnahmen zur Qualitätssteigerung einem institutionalisierten, vom Bund vorgegebenen Prozedere (unter Einbezug und Koordination verschiedener Akteure) folgen. Der Bund formuliert qualitätspolitische Ziele, die sich auf die explizite Setzung von Schwer- punkten und Prioritäten für eine bestimmte Periode fokussieren, und er sorgt dafür, dass für die nachhaltige Umsetzung der Qualitätsstrategie die erforderlichen Mittel bereitgestellt und Strukturen geschaffen werden. Immer wieder wird betont, dass der Bund die Führungsrolle übernehmen will, indem er notwendige Voraussetzungen schafft, Prozesse definiert, eine Rollenklärung herbeiführt - die verschiedenen Akteure sollen klare Rollenzuweisungen erhalten und bei der Umsetzung der Q-Strategie auch entsprechend einbezogen werden - sowie die Handlungsoptionen konkretisiert und Aktionsprogramme definiert. Zur Konkretisierung: Bis Ende März 2010 soll ein detaillierter Projektauftrag durch eine vom Bund eingesetzte Projektleitung definiert werden. Bis Ende 2011 soll die Q-Strategie konkretisiert, eine nationale Organisation zur Koordination der Qualitäts-Aktivitäten aufgebaut werden, dies sowohl im ambulanten wie im stationären Bereich. In der Zwischenzeit sollen die (bislang relativ bescheidenen) Aktivitäten des Bundes fortgesetzt werden. In der Qualitätsstrategie des Bundes sind 9 Aktivitätsfelder definiert: Führung und Verantwortung; Umsetzungskapazitäten/Ressourcen; Information; Anreize, Design Versorgungssystem; Versorgungs- und Begleitforschung; Bildung; Einbezug Patienten und Bevölkerung. Sie werden die Struktur der Q-Strategie bilden. Die Kosten werden auf 20-25 Mio. Franken bei Vollbetrieb der Nationalen Organisation ab 2012 geschätzt, wofür genau wird jedoch nicht weiter ausgeführt. Finanziert werden soll das Ganze allenfalls über Kranken- und Unfallversicherungsprämien, eine Private Public Partnership oder über das reguläre Budget von Bund und Kantonen. Eine Priorisierung wird im Strategiepapier nicht vorgenommen.

Wertung aus Sicht des Ressort DDQ: Nach 11/2 Jahren Warten auf das Strategiepapier steht darin nichts, was man nicht erwartet hätte. Das Ressort hat das meiste antizipiert und ist deshalb nicht überrascht worden. Begrüssenswert ist - und das wird auch wiederholt deklariert -, dass eine Kooperation mit sämtlichen Akteuren wichtig sei. Nachdem das Papier zur Verfügung stand, hat die FMH zuhanden des Bundesrates eine Stellungnahme verfasst. BR Burkhalter hat rasch und in einem sehr offenen und positiv formulierten Brief geantwortet und u.a. versichert, dass das BAG im 1. Quartal 2010 auf die FMH zukommen wird, um Umfang und Art und Weise des Einbezugs der FMH zu besprechen. Ziel der FMH ist, auf den Projektauftrag, der bis Ende März definiert werden muss, Einfluss nehmen zu können. Grundsätzlich begrüssenswert ist die Schaffung des Nationalen Organs, das die verschiedenen Aktivitäten koordinieren soll, insbesondere an der Schnittstelle ambulant/stationär. Begrüsst wird weiter die angestrebte Rollenverteilung und $\mathrm{Zu}$ ständigkeitsregelung und für gut erachtet man, dass im Moment keine Gesetzesänderung hinsichtlich direkter Sanktionen des Bundes gegenüber den Leistungserbringern vorgesehen ist. Positiv zu werten ist schliesslich, dass ein Freiraum besteht, der das Einbringen und 
Weiterführen bestehender Qualitätsaktivitäten ermöglicht. Kritisch zu werten ist der bisherige Einbezug der Akteure seitens des BAG. Als Mitglied der erweiterten Kerngruppe zur Erarbeitung dieser Q-Strategie ergaben sich für die FMH sehr wenig Einflussmöglichkeiten und die Kommunikation der FMH gegenüber verlief mehr als bescheiden: $u$. a. hat die FMH von dieser Qualitätsstrategie erst Stunden nach der Presse erfahren. Bis jetzt stehen Informationen über die Besetzung der Projektleitung aus: Hier wird man versuchen, nachzuhaken und Einfluss geltend zu machen.

Für das Ressort ergeben sich folgende Handlungsfelder: Bereits ist man daran, die Aktionsfelder zu bearbeiten, sich darauf vorzubereiten, in welchen Aktionsfeldern sich die FMH wie, womit und durch wen einbringen soll. Die laufenden Q-Aktivitäten der FMH stehen nicht im Widerspruch zur Qualitätsstrategie des Bundes und werden mit entsprechendem Nachdruck weiter bearbeitet.

Die zweite Aktualität aus dem Ressort DDQ betrifft den von santésuisse und FMH unterzeichneten «Vertrag für die Vereinbarung von Konzepten für die Sicherung und Kontrolle der Medizinischen Leistungserbringung».

Diesem Resultat gingen einige Aktivitäten voraus. Am Beginn stand eine «Arbeitsgruppe Qualität FMH sas», ohne formellen Rahmen. Relativ bald einmal war klar, dass wegen der fehlenden formellen Bedingungen eine gewisse Asymmetrie in der politischen Positionierung dieser Arbeitsgruppe herrschte. FMH-seitig war die Arbeitsgruppe beim ZV, also auf oberster Ebene angesiedelt, die Diskussionspartner jedoch waren innerhalb santésuisse nicht als Entscheidungsträger positioniert. Die FMH löste deshalb im Dezember 2008 die Arbeitsgruppe auf, führte aber die Arbeiten mit dem Projekt Q-Monitoring, der eigentlichen Keimzelle der Zusammenarbeit, innerhalb der FMH weiter. Dies bewog santésuisse, wieder an die FMH heranzutreten

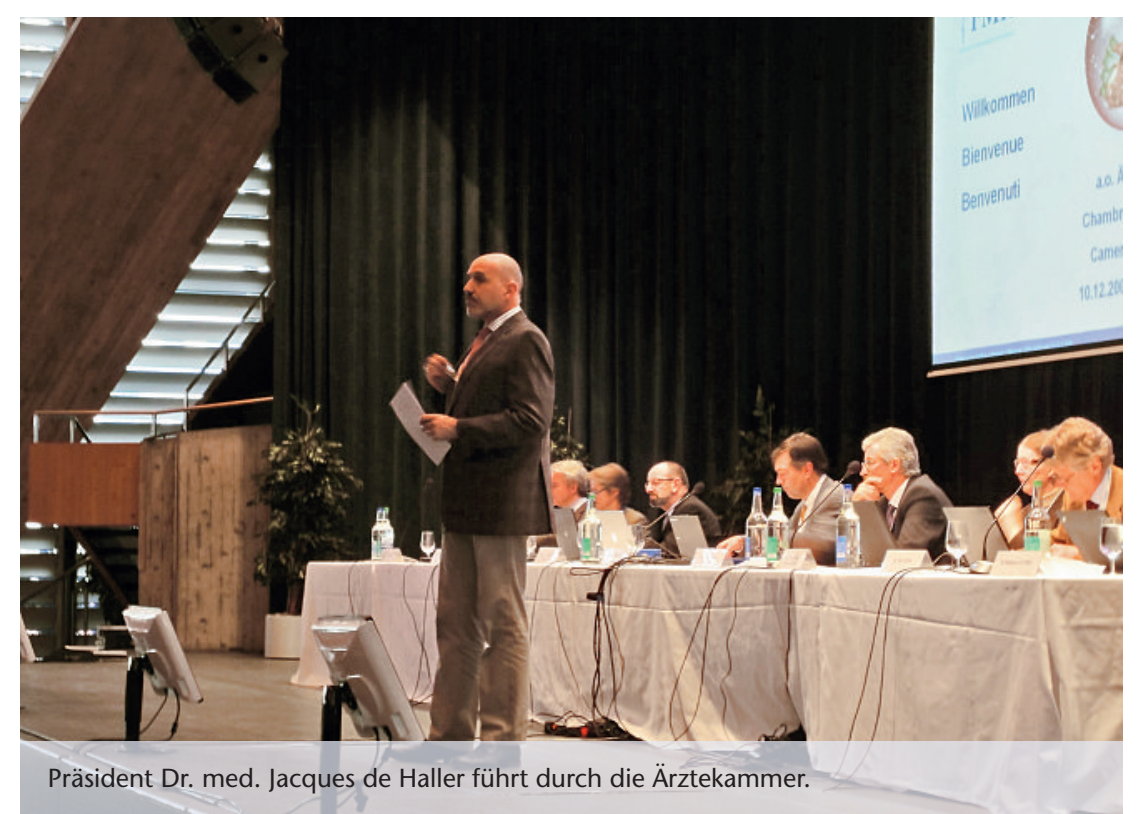

mit dem Wunsch auf Wiederaufnahme der gemeinsamen Arbeiten, diesmal mit Vertretern, die politisch Entscheide fällen können. Der Vorschlag von santésuisse sah ein zweistufiges Vorgehen vor. 1. Stufe: Abschluss eines Grundsatzvertrages, beinhaltend die Regelung der Arbeitsmodalitäten der gemeinsamen Qualitätsgruppe; 2 . Stufe: zusätzliche vertragliche Regelung von konkreten Pilotprojekten. Dieser zwar etwas komplizierte Vorschlag stiess bei der FMH auf Zustimmung, der obenerwähnte Vertrag wurde unterzeichnet.

Der Vertrag erfüllt Art. 58 und Art. 59 KVG sowie Art. $77 \mathrm{KVV}$, mit dem Ziel, Anhang 6 im geltenden TARMED-Rahmenvertrag bezüglich «Regelung über Qualitätserfordernisse und WZW-Kriterien» gemeinsam auszuarbeiten. Die «Arbeitsgruppe Qualität» umfasst je 4 Vertreter von FMH und santésuisse.

Daniel Herren dankt abschliessend seinen Mitarbeiterinnen im Ressort DDQ, einem sehr dynamischen Aufgabenbereich, herzlich für die tolle Zusammenarbeit.

Ch.-A. Favrod-Coune/SVM ist überzeugt, dass viele Ärzte und ärztliche Institutionen in der Schweiz eine Vielzahl von Qualitätsprojekten planen, und stellt die Frage, ob man mit santésuisse bereits über die Finanzierung solcher Pilotprojekte gesprochen habe.

D. Herren/ZV antwortet, dass die erste Sitzung im Januar 2010 stattfinde und der Grundvertrag erst einmal dazu führe, dass man gemeinsam Pilotprojekte definiere. Sollte santésuisse wieder auf das Projekt Q-Monitoring aufspringen wollen, dann muss ganz klar formuliert werden, in welcher Art und Weise die Finanzierung geschehen soll. Sicher wird man innerhalb dieser Arbeitsgruppe Qualität weiter an Anreizmodellen arbeiten. Der Ständerat hat ja grundsätzlich gutgeheissen, für qualitativ gute Arbeit bessere Vertragsmöglichkeiten anzubieten, auch die Politik macht Druck, damit die Finanzierungs- und Anreizfrage geregelt wird.

J. Nadig/SGMO fragt, ob die Begleitforschung auch Teil der Qualitätsstrategie des Bundes sei. D. Herren/ZV antwortet, dass die Begleitforschung zwar namentlich genannt, aber nicht weiter ausdiskutiert sei. Das Papier halte sich diesbezüglich sehr neutral.

Vincenzo Liguori/TI fragt sich, ob sich hinter dem vordergründig gezeigten Interesse von santésuisse an der Qualität der ärztlichen Arbeit nicht vielmehr die Absicht verberge, die Arbeit der Ärzteschaft bzw. deren Wirtschaftlichkeit noch mehr zu kontrollieren.

D. Herren/ZV zeigt sich überzeugt, dass gewisse Kreise bei santésuisse nicht ein wirklich genuines Interesse an der Qualität haben. Daneben gebe es aber genauso Personen, mit welchen man vernünftig diskutieren und gemeinsam das anvisierte Ziel erreichen könne. Man sei sich des Spannungsfeldes absolut bewusst.

Nachdem keine weiteren Fragen gestellt werden, erteilt der Präsident das Wort an Ernst Gähler/ZV für die Informationen aus seinen Ressorts «Paramedics» Sowie «Tarife und Verträge». 
Ressort Paramedics:

\section{MPA neue Bildungsverordnung (BiVo)}

Mit der Schaffung des neuen Berufsbildungsgesetzes im Jahr 2004 wurde der Beruf der MPA zu einem eidgenössischen Lehrberuf. Zwischen 2006 und 2009 hat die Reformkommission/Bildungskommission, präsidiert von Peter Tschudi, die Lehrlingsunterlagen reformiert. Strategisch lag dafür der Lead beim Bundesamt für Bildung und Technologie/BBT, operativ bei der FMH. Eine Organisation der Arbeitswelt/OdA wurde gegründet, in welcher die FMH, die beiden MPA-Verbände SVA und BSMPA sowie die Schulleitungen und die Kantone beteiligt waren.

Während des ganzen Reformprozesses wurden interne und externe Vernehmlassungen durchgeführt.

Die neue Bildungsverordnung wurde im Juli 2009 erlassen, der Vollzug erfolgt auf den 1.1.2010. Die wichtigsten Punkte der neuen Bildungsverordnung MPA: Seit 2004 lautet die Berufsbezeichnung «Medizinische Praxisassistentin EFZ (Eidgenössisches Fähigkeitszeugnis)»; die Lehrdauer beträgt nach wie vor 3 Jahre. Es ist auch weiterhin möglich, mit dem EFZ parallel die Berufsmittelschule zu besuchen, die Schultage sind kantonal geregelt. Das Berufsbild wurde angepasst, die Stärkung der MPA als wichtige Mitarbeiterin gefestigt und die Förderung der Eigenverantwortlichkeit verankert. Die MPA werden vermehrt in Patientenbeziehung, Kommunikation geschult, weiter die Supervision und die Teilnahme an Balint-Gruppen eingeführt. Die untere Alterslimite wurde fallengelassen und die Ausbildungsinhalte gesamtschweizerisch synchronisiert. Mit der Umsetzung der neuen BiVo ab 1.1.2010 hört die Kommissionsarbeit nicht auf: Die Tauglichkeit der Lehrmittel, die Weiterbildungen werden laufend überprüft und entsprechend angepasst. Damit befasst sich die Kommission für Berufsentwicklung, die voraussichtlich weiterhin von Peter Tschudi präsidiert werden wird. Weitere Informationen über die Reform der Bildungsverordnung findet man unter den folgenden Internetadressen: www.fmh.ch/ Dienstleistungen/MPA oder www.mpaschweiz.ch. Im Januar wird die neue BiVo MPA in der SÄZ vorgestellt.

(An dieser Stelle werden die Informationen aus dem ZV-Ressort Gesundheitsförderung und Prävention vorgezogen. Die Informationen aus dem Ressort Tarife und Verträge werden an den Schluss der Sitzung verschoben.)

\section{Tarife und Verträge}

Ernst Gähler/ZV-Ressortleiter informiert im Folgenden die Ärztekammer über diverse Punkte aus seinem Ressort.

- Revision Tarifstruktur TARMED:

Das Projekt 2010 wird zurzeit durch santésuisse blockiert mit einer detaillierten Kostensteuerungsvereinbarung, die sie abgeschlossen haben möchte, bevor das Projekt überhaupt gestartet ist. Bisher hat santésuisse zwei Vorschläge unterbreitet, von denen keiner akzeptabel war. Anlässlich eines Gesprächs mit santésuisse, an welchem neben der
FMH (E. Gähler) auch Urs Stoffel/ZH als Vertreter der KKA teilnahm, konnte ärzteseitig ein konstruktiver Vorschlag eingebracht werden (Vereinfachung der Revision der Tarifstruktur; Kostenmodelle, KoReg für den ambulanten Bereich, INFRA für den stationären Bereich, mit aktuellen Daten wie Löhne, Mieten usw. anpassen). Knackpunkt im Ganzen ist, dass zunächst die Tarifstruktur in Ordnung gebracht werden soll, in einer zweiten Phase dann die Tarifierung. Zu den Eckpunkten in der Tarifstruktur-Revision gehören auch die Besserstellung der Grundversorger (nur möglich, wenn ein eigenes Kapitel für Grundversorger geschaffen wird), die Justierung (Taxpunktwert = 1 Franken), die Tarifierung (im KVG-Bereich über die LeiKoV). Die FMH-Vertretung hat klar deponiert, dass die Revision der Tarifstruktur nicht kostenneutral erfolgt, sondern eine Steigerung möglich sein muss. Inzwischen begrüsst santésuisse als Verband dieses Modell sowohl auf der politischen wie auf der technischen Ebene, innerhalb von santésuisse muss dies nun aber noch mit den verschiedenen Versicherern besprochen und abgestimmt werden. Im Leitungsgremium TARMED Suisse wurde unser Vorschlag ebenfalls als Grundlage für die weitere Arbeit akzeptiert.

- Revision Dignitätskonzept:

Das aktuell noch geltende Dignitätskonzept 9.0, seinerzeit mit dem TARMED genehmigt, weist sehr grosse Mängel auf. Es wurde deshalb beschlossen, dieses Dignitätskonzept zu revidieren. An die Stelle der Besitzstandsfortbildung sollen die Fortbildungsdiplome (Fortbildungsbestätigungen für Nicht-FMH-Mitglieder) treten. Dies entspricht einer liberalen, sehr viel einfacheren Lösung und ist für die Ärzteschaft ein grosser Fortschritt. E. Gähler verweist auf den Artikel von Christoph Hänggeli/FMH, der letzte Woche in der SÄZ erschienen ist. In einer zweiten Phase geht es dann darum, die qualitativen Dignitäten ebenfalls zu vereinfachen und so auszugestalten, dass auch die jungen Kollegen, die keine Besitzstände geltend machen können, medizinische Leistungen ausserhalb ihres Facharzttitels werden abrechnen können, sofern sie über die entsprechende Weiterund Fortbildung verfügen.

- Analysenliste:

Die Firma INFRAS hat für das Monitoring ein Konzept erstellt und führt dieses nun durch. Alle unsere Intentionen konnten in das Konzept eingebracht werden, ausser, dass die Analysenliste sachgerecht gerechnet ist. Diese Tatsache berechtigt uns - dies wurde auch seitens des BAG bestätigt - ein erweitertes Monitoring durchzuführen. Knackpunkt hier ist die Vereinbarung zur Datenlieferung. Das BAG kann das Monitoring ohne Daten der Ärzteschaft nicht durchführen. Wir fordern ein Mitspracherecht beim Schlussbericht bzw. bei der Interpretation der Resultate und wir wol- 
len eine finanzielle Abgeltung für unsere Mehraufwendungen. P. Indra und Th. Zeltner vom BAG, bei welchen unsere Forderung momentan liegt, haben bestätigt, dass etwas zu machen ist. Die FMH hat klargemacht, dass keine Daten fliessen werden, bevor diese Vereinbarung steht.

Im erweiterten Monitoring möchten wir die ganze Analysenkette abbilden, denn ganz entscheidend ist, auch die Daten der Auftragslaboratorien zu haben. Wir haben Daten von der Ärztekasse, von NewIndex (aus den Trustcenter und der Nationalen Konsolidierungsstelle NAKO), aus dem Qualitätskontrollzentrum und von Privatlabors für diejenigen Ärzte, die uns mittels Talon autorisiert haben, ihre Daten dort abzuholen. Mittlerweile sind es gut 1600 Ärztinnen und Ärzte, die ihre Daten zur Verfügung stellen. E. Gähler ruft die Delegierten dazu auf, ihre Kolleginnen und Kollegen zu animieren, den Talon, der zweimal in der Ärztezeitung erschienen ist und von verschiedenen Kantonalen Ärztegesellschaften mit Mitteilungen an ihre Mitglieder verschickt wurde, auszufüllen und einzuschicken. Diese Daten sind ganz entscheidend. Das erweiterte Monitoring wird weiter eine differenzierte Auswertung zulassen: Die Auswirkungen im Bereich des Praxislabors werden bezüglich der sachgerechten Berechnung gut dargestellt werden können, ebenso die Auswirkungen auf die LeiKoV - für die Tarifierung der Taxpunktwerte in den nächsten Jahren nicht unwesentlich - sowie auch die Auswirkung auf den TARMED selbst. Seit dem 1.7.09 ist die neue Analysenliste in Kraft. Die bisherigen Auswertungen haben alle unsere Simulationen und Aussagen, die wir bereits vor der Einführung der neuen AL gemacht und dem BAG gegenüber detailliert ausgeführt hatten, bestätigt. Die Verlierer sind die Allgemeinpraktiker und die Allgemein Internisten, bei welchen der Laborumsatz pro Sitzung um 25\% gesunken ist. Die Ärzteschaft hat sich, statistisch gesehen, bisher vorbildlich verhalten, sie konzentriert sich auf eine qualitativ gute Betreuung ihrer Patienten, was die ihr von verschiedenen Seiten permanent unterstellten pekuniären Interessen klar in Abrede stellt.

- Taxpunktwertverhandlungen mit der MTK:

Hier gibt es nur wenig Neues zu berichten. Wenn die Kostensteuerungsvereinbarung mit santésuisse unterzeichnet werden kann, gibt es eine Taxpunktwertanhebung von 1 Rappen, die restlichen 3 Rappen, die wir zugute haben, würden in die Strukturrevision einfliessen und uns dort gutgeschrieben. Das Problem ist hier wieder das Junktim mit der Tarifrevision 2010, und dass sich santésuisse nicht bewegt. Möglicherweise wird die MTK am 15. Dezember 2009 aber etwas anderes beschliessen. Im Moment wird hier unsererseits weiter gearbeitet, weitere allenfalls zu prüfende Massnahmen wurden in der Delegiertenversammlung diskutiert.
- Ärztliche Medikamentenabgabe DMA/SD:

Hier läuft der Kampf auf zwei Ebenen: Der Bund möchte, mit zum Teil sehr eigenartigen, politisch schwierig nachvollziehbaren Begründungen, über die Revision des Heilmittelgesetzes die ärztliche Medikamentenabgabe abschaffen. Die Vernehmlassung läuft bis zum 5. Februar 2010. Die DV hat einstimmig, mit wenigen Enthaltungen, beschlossen, die ärztliche Medikamentenabgabe zu unterstützen, auch die welschen Kollegen unterstützen dies. Eine Taskforce mit je einem Vertreter des VEDAG, der FMH, der KKA, der HÄCH und der APA wurde eingesetzt und ist daran, Strategien zu entwickeln.

Die zweite Ebene ist die Absenkung der Margen: Bundesrat Couchepin hatte verkündet, die Vertriebsmarge von $15 \%$ auf $12 \%$ bereits auf den 1.3.2010 zu senken, was für die Kantone mit ärztlicher Medikamentenabgabe eine deutliche Einbusse bedeutet. Die ständerätliche Gesundheitskommission hat im Parlament eine Absenkung gar auf $8 \%$ gefordert, was glücklicherweise in beiden Räten abgelehnt wurde. Hängig ist noch die Motion Diener/ZH, welche eine differenzierte Marge zwischen Abgabekanal Apotheke und Abgabekanal Patientenapotheke einführen möchte. Die Motion ist an den Bundesrat überwiesen worden. Hier ist seitens FMH eine schon länger eingesetzte Arbeitsgruppe daran, neue Modelle zu finden, damit wir von diesen Margen unabhängig werden.

E. Gähler/ZV dankt zum Schluss seiner Ausführungen allen Kolleginnen und Kollegen, die in den diversen Kommissionen mitarbeiten, herzlich für ihre Unterstützung und bedankt sich ebenso herzlich bei seinen Mitarbeitenden im FMH-Tarifdienst/Bereich Tarife und Verträge, die, mit Irène Marty an der Spitze, eine grosse Arbeit leisten.

\section{Weitere Mitteilungen}

Christine Romann/ZV-Ressort Gesundheitsförderung und Prävention macht die Delegierten zunächst kurz auf drei Artikel aufmerksam, welche Projekte aus dem Ressort darstellen. Die Artikel wurden veranlasst, um Ärztinnen und Ärzten aufzuzeigen, welche Projekte in ihren eigenen Reihen laufen.

Der erste, bereits in der SÄZ (Nr. 45/2009) erschienene Artikel befasste sich mit dem Projekt des KHM «Gesundheitscoaching - ein Programm für Gesundheitsförderung und Prävention in der Hausarztpraxis». Der zweite Artikel betrifft das Projekt «EviPrev, ein Programm zur Prävention und Gesundheitsförderung in der Hausarztpraxis». Er wird in einer der nächsten SÄZ-Ausgaben erscheinen. Der dritte Artikel betrifft das Projekt «dialog-gesundheit - neue hausärztliche Public Health». Auch dieser Artikel wird demnächst in der SÄZ publiziert werden.

Danach geht C. Romann/ZV etwas eingehender auf das Präventionsgesetz ein. Sie erinnert eingangs kurz 
an den Zweckartikel in den FMH-Statuten, wo u.a. steht, dass «die FMH zur Gesundheitsförderung beitragen will». Diese Absicht bildet die statutarischen Grundlagen der Arbeiten in ihrem Ressort, gleichzeitig auch der Arbeiten im Zusammenhang mit dem Präventionsgesetz. Begrüsst wird das Gesetz insbesondere, weil es eine gesetzliche Grundlage für die nicht übertragbaren psychischen und physischen Krankheiten liefert und weil es wichtige gesundheitsfördernde Grundsätze verankert - beispielsweise das Ziel, soziale Ungleichheiten abzubauen. Wichtig ist dabei auch die Gesundheitsfolgenabschätzung, d.h. jedes Geschäft, das dem Parlament unterbreitet wird, soll hinsichtlich seiner Folgen auf die Gesundheit der Bevölkerung überprüft werden. Das Gesetz will die Bevölkerung nicht bevormunden, sondern es bleibt beim Individuum, sich um seine Gesundheit zu kümmern. Schliesslich bringt das Gesetz die Einführung nationaler Ziele, eine bundesrätliche Strategie und die Gesundheitsberichterstattung, als Steuerungsinstrumente. Das Gesetz legt die Rollenverteilung unter den verschiedenen Akteuren fest und sieht ein Schweizerisches Institut für Prävention und Gesundheitsförderung vor. Letzteres wird unterschiedlich beurteilt: Während es von den einen als Verschlankung der Strukturen befürwortet wird, sehen andere darin einen Wasserkopf, sozusagen ein zweites BAG. Abschliessend zeigt C. Romann/ZV anhand zweier Folien die aktuelle Organisation und Steuerung und die neue Steuerung der Mittelverwendung, wie sie im Präventionsgesetz geplant ist. Die finanziellen Mittel (Bundesmittel, Tabakpräventionsabgabe und Zuschlag auf KVG-Prämien) sollen über die Bundesstellen und das geplante Institut fliessen. Letzteres verteilt die Mittel z.B. an kantonale Programme und Projekte, an Projekte von Gesundheitsligen und NGOs sowie an Projekte von Gesundheitsberufen und -institutionen. Mit dem Gesetz wird es künftig klarer ersichtlich sein, welche finanziellen Mittel zu welchem Zweck wohin fliessen. C. Romann/ZV schliesst ihre Informationen in der Hoffnung, das Gesetz überstehe erst einmal die Eintretensdebatte, komme danach im Sommer 2010 in die Räte und werde - mit oder ohne Institut - seinen Weg in die Gesetzgebung finden.

Ch. Bossard/VSAO stellt an dieser Stelle den Ordnungsantrag, solange das erforderliche Quorum noch gegeben sei, die Beschlusstraktanden vorzuziehen. Dem Ordnungsantrag wird mit grosser Mehrheit, bei wenigen Gegenstimmen, stattgegeben.

\section{Budgetnachtrag für das Jahr 2010}

Der Präsident führt in das Thema ein. Die EMH ist eine Aktiengesellschaft, an welcher die FMH mit 55\%, der Schwabe Verlag mit $45 \%$ beteiligt ist. Im Verwaltungsrat sitzen als FMH-Delegierte Ludwig Th. Heuss (VRP), Hans Kurt und Jean Martin. Das Kerngeschäft der EMH umfasst 3 Pfeiler, welche, gemäss dem Zweckartikel der FMH, die FMH-Politik, die Fortbildung und die Forschung abdecken: die Schweizerische Ärztezeitung/ SÄZ (Politik), das Swiss Medical Forum (Fortbildung) und das Swiss Medical Weekly (Forschung). Die EMH verfolgt mit diesen drei Publikationen eine offene Redaktionspolitik und eine sowohl im politischen wie auch wirtschaftlichen Bereich strikte redaktionelle Unabhängigkeit. Die Medienbranche weltweit ist von der Finanzkrise stark getroffen worden, wovon auch die EMH nicht verschont blieb. Das Inseratevolumen ist seit Ende 2008 derart stark zurückgegangen, dass nach Einschätzung der EMH-Unternehmensleitung ohne Fremdhilfe mittel- und langfristig die Herausgabe der SÄZ und der weiteren Printprodukte nicht mehr garantiert werden kann. Um die Situation nicht schon im 2009 eskalieren zu lassen, insbesondere die Herausgabe der SÄZ nicht zu gefährden, hat der Zentralvorstand nach mehreren Gesprächen mit der EMH beschlossen, zulasten der Rechnung 2009 einen Unterstützungsbeitrag von CHF 15.00 pro FMH-Mitglied, rund CHF 0,5 Mio., zu sprechen. Die GPK, die ebenfalls Gespräche mit EMH führte, hat dieses Vorgehen gutgeheissen. Die jüngsten Gespräche mit EMH haben allerdings gezeigt, dass sich die prekäre Situation bezüglich Inserateeinnahmen weder entschärft hat noch mittelfristig entschärfen dürfte. Umfangreiche Kosteneinsparungs- und Effizienzsteigerungsmassnahmen wurden in den letzten Jahren durch EMH getroffen. Nachdem aber diese Massnahmen nicht ausreichen, um die Herausgabe der erwähnten Printmedien der EMH mittel- bis langfristig zu sichern, bleibt nach Einschätzung des ZV und der GPK als einzige realistische Massnahme, die zudem rasch und einfach umgesetzt werden kann, die Printmedien der EMH mit einer moderaten Abo-Gebühr seitens der FMH-Mitglieder zu unterstützen.

Der Ärztekammer liegt ein Antrag des Zentralvorstands auf Einführung einer Abo-Gebühr ab 2010 in der Höhe von CHF 30.00 pro Jahr für alle FMH-Mitglieder, mit Ausnahme der Mitgliederkategorie 4/Ärztinnen und Ärzte in Weiterbildung, vor. Damit soll jedes FMH-Mitglied weiterhin je eine Printausgabe der SÄZ und des Swiss Medical Forum, den Zugang zu deren elektronischen Fassungen sowie des Swiss Medical Weekly erhalten.

Der Präsident eröffnet anschliessend die Diskussion. Hans Kurt/FMPP und VR EMH unterstreicht, dass nur die drei erwähnten Publikationen (SÄZ, Swiss Medical Forum, Swiss Medical Weekly) finanziell unterstützt werden. Alle übrigen Produkte der EMH müssen sich selbst finanzieren. $R$. Schwarz/GPK bestätigt, dass die beantragten 30 Franken fürs Erste genügten, dass der ZV aber aufgerufen sei, bei der EMH eine nachhaltige Situation herbeizuführen. Jean-Pierre Pavillon/VD bezieht sich auf seinen Antrag Nr. 2 zu Traktandum 6, in welchem er fordert, «dass der Beschluss über die Gewährung einer finanziellen Unterstützung an die EMH vertagt werde, bis eine detaillierte Analyse der Rentabilität der verschiedenen Publikationen der EMH vorliegt». Seiner Ansicht nach - und er wird darin von verschiedenen Vertretern aus der Romandie unterstützt -, ist einzig die SÄZ unverzichtbar und verdient somit als einzige Publikation die Unterstützung aller FMH-Mitglieder. 
Nur wenn aber belegt werden könne, dass die SÄZ für sich allein genommen defizitär sei, wäre ein finanzieller Beitrag an EMH akzeptabel.

Alexander von Weymarn/TG bringt noch einen weiteren Aspekt in die Diskussion ein: Wenn schon bezahlt werden müsse, dann solle auch ein gewisser Einfluss auf die Inserate möglich sein, und erwähnt als Beispiel das Inserat der medidata. M. Müller/KHM bittet die Kammer, jetzt nicht das Kind mit dem Bade auszuschütten. Bis jetzt hätten alle drei erwähnten Zeitschriften ihren Erscheinungsgrund, gerade das Swiss Medical Forum sei beispielsweise eine vielgelesene Fortbildungszeitschrift. Alle seien Opfer der aktuellen Wirtschaftslage, es sei eine Illusion zu glauben, man könne werbefreie wissenschaftliche Zeitschriften herstellen. Im Sinne eines abwartenden Verhaltens plädiert er für die finanzielle Unterstützung der EMH.

Er findet Unterstützung bei Ch. Ramstein/VEDAG, welcher seinen Antrag Nr. 3 zu Traktandum 6 erläutert: Entgegen der Vermutung der meisten FMH-Mitglieder fliesse bislang kein Franken aus den Mitgliederbeiträgen in die Produktion der SÄZ. Die Produktionskosten werden ausschliesslich durch Einnahmen aus der gemeinsamen Inserate-Akquisition gedeckt. Die Druckversuche der inserierenden Firmen, auf den Inhalt der Zeitschriften direkt Einfluss zu nehmen, hätten massiv zugenommen, die Qualität und Unabhängigkeit der ärzteeigenen Informationsorgane sei gefährdet. Der VEDAG beantragt deshalb, «die EMH im 2010 mit einem einmaligen Sonderbeitrag von CHF 30.00 pro FMHMitglied (FMH-Mitglieder Kat. 4 ausgenommen) finanziell zu unterstützen. Dieser Sonderbeitrag sei neben den mit dem Budget 2010 bereits genehmigten Mitgliederbeiträgen zu erheben. Gleichzeitig soll der $Z V$ beauftragt werden, zusammen mit der EMH bis zur ord. Ärztekammer im Mai 2010 das bisherige Geschäftsmodell den aktuellen und zukünftigen Gegebenheiten anzupassen und ein Konzept für eine nachhaltige Finanzierung zu entwickeln, welche die Unabhängigkeit und Qualität der für die gesamte Ärzteschaft wichtigen Publikationsorgane sicherstellen. Dies sei an der Ärztekammer im Mai 2010 vorzulegen».

Ch.-A. Favrod-Coune unterstreicht die kritische Haltung der Romandie mit dem Hinweis auf die Revue Médicale Suisse, einem wichtigen wissenschaftlichen Organ in der Romandie, die ebenfalls Probleme habe. Er befürchtet, in einigen Monaten könnten auch die Romands mit einem entsprechenden Unterstützungsantrag für ihr eigenes Organ kommen. Er ruft deshalb dazu auf, dass EMH und FMH das Geschäftsmodell, was die drei Zeitschriften betreffe, überdenkt.

H. Kurt/FMPP und VR EMH präzisiert, dass die drei Zeitschriften zusammen beworben und gemeinsam verschickt würden. Im Swiss Medical Forum seien immerhin zu 20\% französische Original-Artikel abgedruckt, und das Swiss Medical Weekly sei online zugänglich, eine Abo-Gebühr werde nur für die Printausgabe erhoben. Franco Denti/OMCT weist darauf hin, dass auch das Tessin ein eigenes Organ hat. R. Schwarz/ GPK erinnert daran, dass die finanzielle Situation der EMH in der Vergangenheit eine ganz andere war, die
FMH in den letzten Jahren über 9 Mio. Franken an Lizenzgebühren eingenommen habe. Hans-Anton Vogel/AI, AR erkundigt sich, ob der ZV nicht an eine Reinvestition der Lizenzgebühren gedacht habe? Der Präsident antwortet, dass man aus Transparenzgründen nicht einfach auf die Lizenz habe verzichten wollen.

Roland Oppliger/SGAI stellt an dieser Stelle den Ordnungsantrag, jetzt unverzüglich zur Abstimmung zu schreiten.

Der Ordnungsantrag wird einstimmig angenommen.

Der Präsident lässt zunächst über den Antrag J.-P. Pavillon/VD Nr. 2 zu Traktandum 6 abstimmen. Die Ärztekammer lehnt diesen Antrag mit 58 zu 54 Stimmen $a b$.

Daraufhin erklärt der Präsident, nach Rückversicherung beim Zentralvorstand, dass dieser sich dem Antrag des VEDAG anschliessen könne.

Die Ärztekammer genehmigt anschliessend den Antrag Ch. Ramstein/VEDAG Nr. 3 zu Traktandum 6 mit klarer Mehrheit, bei einigen Enthaltungen.

\section{Position Managed Care der FMH}

Der Antrag Nr. 1 zu Traktandum 7 wurde eingangs der Sitzung als eigenes, beschlussfähiges Traktandum in die Traktandenliste aufgenommen.

Ch. Bosshard/VSAO erläutert den Antrag: Der politische Entscheidfindungsprozess schreitet nun rasch voran. Die Sicherheits- und Gesundheitskommission des Nationalrats entscheidet im Januar 2010, das Plenum des Nationalrats eventuell bereits in der Frühjahrssession. Der VSAO vertritt die Meinung, die FMH solle anhand der aktuellen Diskussionen ihre Positionen überprüfen, da nach wie vor unter anderem Listenmodelle der Kassen drohten. Der Ärztekammer wird deshalb beantragt, «die Delegiertenversammlung (DV) zu beauftragen, die verschiedenen aktuell existierenden und zur Diskussion stehenden Modelle zur Förderung der integrierten Versorgung vertieft zu prüfen, zu diskutieren, und deren Vor- und Nachteile aufzuzeigen. Insbesondere soll die bisherige Position der FMH im Kontext des Nationalrats-Modells, des Modells des vpod Persönliche Gesundheitsstelle/PGS sowie des Visana-Modells geprüft werden».

Nachdem das Wort nicht verlangt wird, bringt der Präsident den Antrag zur Abstimmung.

Die Ärztekammer genehmigt den Antrag Ch. Bosshard/VSAO Nr. 1 zu Traktandum 7 einstimmig, bei einigen Enthaltungen.

(An dieser Stelle folgen die Informationen aus dem Ressort Tarife und Verträge)

\section{Varia}

Das Wort zu Varia wird nicht verlangt.

Der Präsident Jacques de Haller gratuliert zum Abschluss dem neu gewählten SIWF-Präsidenten Werner Bauer nochmals herzlich zur Wahl und bedankt sich bei P.-F. Cuénoud für sein Engagement. Die Delegierten unterstreichen dies mit herzlichem Applaus.

Punkt 17.30 Uhr schliesst der Präsident die ausserordentliche Sitzung der Ärztekammer und entlässt die Delegierten mit den besten Wünschen. 


\section{Glossar der ausserordentlichen Ärztekammer vom 10.12.2009}

\begin{tabular}{|c|c|c|c|}
\hline $\begin{array}{l}\text { APA } \\
\text { BiVo }\end{array}$ & $\begin{array}{l}\text { Ärzte mit Patientenapotheke } \\
\text { Bildungsverordnung }\end{array}$ & SGAI & $\begin{array}{l}\text { Schweiz. Gesellschaft für Allergologie } \\
\text { und Immunologie }\end{array}$ \\
\hline BSMPA & $\begin{array}{l}\text { Bund Schweizer Verbände Medizi- } \\
\text { nischer Praxis-Assistentinnen }\end{array}$ & SGG & $\begin{array}{l}\text { Schweiz. Gesellschaft für Gastro- } \\
\text { enterologie }\end{array}$ \\
\hline $\begin{array}{l}\text { DDQ } \\
\text { DMA }\end{array}$ & $\begin{array}{l}\text { Daten, Demographie, Qualität } \\
\text { Direkte Medikamentenabgabe }\end{array}$ & SGH & $\begin{array}{l}\text { Schweiz. Gesellschaft für Hand- } \\
\text { chirurgie }\end{array}$ \\
\hline $\begin{array}{l}\text { DRG } \\
\text { DV }\end{array}$ & $\begin{array}{l}\text { Diagnosis Related Groups } \\
\text { Deleqiertenversammlung }\end{array}$ & SGIM & $\begin{array}{l}\text { Schweiz. Gesellschaft } \\
\text { für Innere Medizin }\end{array}$ \\
\hline $\mathrm{nCh}$ & $\begin{array}{l}\text { Foederatio medicorum } \\
\text { chirurgicorum helvetica }\end{array}$ & SGMO & $\begin{array}{l}\text { Schweiz. Gesellschaft } \\
\text { für Medizinische Onkologie }\end{array}$ \\
\hline MPP & $\begin{array}{l}\text { Foederatio Medicorum Psychiatri- } \\
\text { corum et Psychotherapeuticorum }\end{array}$ & SGKPT & $\begin{array}{l}\text { Schweiz. Gesellschaft für Klinische } \\
\text { Pharmakologie und Toxikologie }\end{array}$ \\
\hline $\begin{array}{l}\text { GPK } \\
\mathrm{HÄCH}\end{array}$ & $\begin{array}{l}\text { Geschäftsprüfungskommission } \\
\text { Berufsverband der Haus- }\end{array}$ & SGPP & $\begin{array}{l}\text { Schweiz. Gesellschaft für Psychiatrie } \\
\text { und Psychotherapie }\end{array}$ \\
\hline INFRA & $\begin{array}{l}\text { und Kinderärzte Schweiz } \\
\text { Infrastrukturkosten }\end{array}$ & SIWF & $\begin{array}{l}\text { Schweiz. Institut für medizinische } \\
\text { Weiter- und Fortbildung }\end{array}$ \\
\hline KA & $\begin{array}{l}\text { Konferenz der Kantonalen Ärzte- } \\
\text { gesellschaften }\end{array}$ & SRO & $\begin{array}{l}\text { Schweiz. Gesellschaft für Radio- } \\
\text { Onkologie }\end{array}$ \\
\hline oReg & Kostenrechnung & SVA & Schweiz. Verband Medizinischer \\
\hline LeiKoV & Leistungs- und Kosten-Vereinbarung & & PraxisAssistentinnen \\
\hline NGO & $\begin{array}{l}\text { Nicht-staatliche Organisationen } \\
\text { (Non-Governmental Organisations) }\end{array}$ & VEDAG & $\begin{array}{l}\text { Verband Deutschschweizerischer } \\
\text { Ärztegesellschaften }\end{array}$ \\
\hline $\begin{array}{l}\text { OdA } \\
\text { OMCT }\end{array}$ & $\begin{array}{l}\text { Organisation der Arbeitswelt } \\
\text { Ordine dei medici del Cantone Ticino }\end{array}$ & vpod & $\begin{array}{l}\text { Verband des Personals Öffentlicher } \\
\text { Dienste }\end{array}$ \\
\hline SÄZ & Schweizerische Ärztezeitung & VR & Verwaltungsrat \\
\hline SAMM & $\begin{array}{l}\text { Schweizerische Ärztegesellschaft } \\
\text { für manuelle Medizin }\end{array}$ & $\begin{array}{l}\text { VRP } \\
\text { VSAO }\end{array}$ & $\begin{array}{l}\text { Präsident des Verwaltungsrats } \\
\text { Verband Schweizerischer Assistenz- }\end{array}$ \\
\hline $\begin{array}{l}\text { SD } \\
\text { SFSM }\end{array}$ & $\begin{array}{l}\text { Selbstdispensation } \\
\text { Swiss Federation of Specialities } \\
\text { in Medicine }\end{array}$ & & und Oberärztinnen und -ärzte \\
\hline
\end{tabular}

